\title{
Ulcerative colitis is characterized by amplified acute inflammation with
}

\section{delayed resolution}

Riccardo Wysoczanski ${ }^{1}$, Alexandra C Kendall ${ }^{2}$, Madhur Motwani ${ }^{3}$, Roser Vega ${ }^{4}$, Farooq Z Rahman $^{4}$, Sara McCartney ${ }^{4}$, Stuart L Bloom ${ }^{4}$, Anna Nicolaou ${ }^{2}$, Derek W Gilroy ${ }^{3}$, Anthony W Segal ${ }^{1}$, Daniel J B Marks ${ }^{1}$

${ }^{1}$ Centre for Molecular Medicine, University College London, London, UK; ${ }^{2}$ Laboratory for Lipidomics and Lipid Biology, Division of Pharmacy and Optometry, School of Health Sciences, Faculty of Biology, Medicine and Health, University of Manchester, Manchester Academic Health Science Centre, UK; ${ }^{3}$ Centre for Clinical Pharmacology and Therapeutics, University College London, London, UK; ${ }^{4}$ Department of Gastroenterology, University College London Hospital, London, UK.

Correspondence to Professor Anthony W Segal, Centre for Molecular Medicine, Rayne Building, 5 University Street, London WC1E 6JJ, UK. Tel: +44 207679 6173. E-mail: t.segal @ucl.ac.uk

Short running title: Inflammation in Ulcerative colitis 


\section{Abstract}

The cause of chronic inflammation in ulcerative colitis (UC) is incompletely understood. Here we tested the hypothesis that an excessive acute inflammatory response to bacteria contributes to the pathogenesis. Acute inflammatory responses were provoked in vivo in UC patients and healthy controls by intradermal inoculation with bacteria. Vascular responses were quantified by laser Doppler. Inflammatory exudates were recovered in superimposed suction blisters and cells measured by polychromatic flow cytometry, cytokines by multiplex array, and inflammatory lipids by mass spectrometry. Vascular responses in UC patients were heightened at $24 \mathrm{~h}$ after bacterial injection $(\mathrm{p}=0.03)$, and remained abnormally high at $48 \mathrm{~h}(\mathrm{p}=0.0005)$ and this amplified response was seen in UC with Gram-positive as well as Gram-negative organisms $(\mathrm{p}=0 \cdot 01)$. The cellular infiltrate over the injection site, composed largely of neutrophils at 4 hours a was greater in $\mathrm{UC}(\mathrm{p}=0 \cdot 002)$. At $48 \mathrm{~h}$, the increased numbers of cells in UC were composed of neutrophils $(\mathrm{p}=0 \cdot 001)$ and CD4 lymphocytes $(\mathrm{p}=0 \cdot 001)$. The exaggerated inflammation in UC was not a cytokine-driven phenomenon. Exaggerated onset was normalised in patients taking 5aminosalicylates, accompanied by increased concentrations of hydroxy fatty acids 9-oxooctadecadienoic acid (OxoODE; $\mathrm{p}=0 \cdot 05)$ and 13-OxoODE $(\mathrm{p}=0 \cdot 01)$ in resolving exudates. In vitro, these compounds suppressed macrophage inflammatory cytokine secretion through PPAR $\gamma$ $(\mathrm{p}<0 \cdot 0001)$. Conversely, 5-aminosalicylates did not inhibit early inflammatory reactions in control participants. Acute inflammatory responses to bacteria in UC are both overly exuberant and slow to resolve. Neutrophils accumulate in excess and persist, in keeping with the pathological appearances of disease flares. These studies also provide new insight into the mechanism of 5-aminosalicylate (5ASA) drugs, which act as pro-resolution rather than indiscriminate anti-inflammatory agents by promoting formation of immunomodulatory hydroxy 
lipids. While production of these lipids is not defective as part of the underlying disease process, this identifies a novel mechanism of drug action harnessing pro-resolution pathways.

\section{Summary}

Wysoczanski and colleagues demonstrate that the inflammatory response to injected bacteria is exaggerated and prolonged in ulcerative colitis. This disordered inflammation appears to be associated with increased secretion of PGE2. 5-aminosalicylate drugs, which are used to treat this condition, normalize inflammation and PGE2 secretion, and appear to work through PPAR $\gamma$ 


\section{Introduction}

Ulcerative colitis (UC) is characterized by chronic inflammation, principally affecting the large bowel, but with potential to involve skin, eyes and joints (Ungaro et al., 2017). The incidence is 6 $3-24 \cdot 3$ per 100,000 person-years (Molodecky et al., 2012), with considerable associated morbidity (including a 30\% lifetime risk of surgery and predisposition to colorectal cancer. Eaden et al, 2001). The underlying cause of UC remains unclear, and theories focus on perturbations in the immune system, intestinal barrier or gut contents (including microbiota). Genome-wide association studies (GWAS) have identified over 160 susceptibility loci for inflammatory bowel disease (de Souza and Fiocchi, 2016). Candidate genes have roles in immune responses and mucosal barrier integrity, although mechanistic consequences remain broadly speculative (McGovern et al., 2015). Existing therapies for UC principally target TNF- $\alpha$, T cells (thiopurines, calcineurin inhibitors and integrin blockers) or janus kinase; however, all have pleiotropic downstream effects, and a substantial proportion of patients do not achieve complete remission and mucosal healing (Ungaro et al., 2017; Verstockt et al., 2018).

An additional aetiological component in UC could be that acute inflammation is excessive. Neutrophils are prominent in the colonic mucosa during flares of UC, and we have previously demonstrated that UC patients mount protracted early inflammation following challenge with E. coli (Marks et al., 2006a; Marks et al., 2006b). This could reflect the situation in the large bowel, where there are approximately $10^{12}$ bacteria/gram of colonic contents and Gram-negative species predominate. While considerable effort has gone into characterizing the pathways and molecules that drive the initiation of inflammation, much less is known about its termination and resolution (Gilroy and De Maeyer, 2015). It is now evident that there are active mechanisms that promote resolution, including clearance of the initiating stimulus, scavenging of 
cytokines, neutrophil apoptosis, and phagocytosis of effete neutrophils by macrophages. If

effective, this coordinated sequence of events prevents the inflammation becoming chronic. We therefore performed a unique set of in vivo experiments to delineate the nature of aberrant acute inflammatory responses in UC and to identify the underlying causal processes.

\section{Results}

\section{Blood flow in response to $E$. coli}

We examined acute inflammatory responses following inoculation with UV-killed $E$. coli

(UVkEc) in 13 healthy controls (HC), 10 UC patients on no treatment (UC), and 11 UC patients receiving 5ASA drugs (UC-5ASA).

In $\mathrm{HC}$, vascular responses were maximal at $24 \mathrm{~h}$, and almost completely resolved within $48 \mathrm{~h}$ (figure 1A,D). In UC patients not receiving 5ASA drugs, blood flow attained higher peaks $(p=0.03)$, and the rate of change between $24 \mathrm{~h}$ and $48 \mathrm{~h}(\mathrm{p}=0.03$; figure $1 \mathrm{~B}, \mathrm{D})$ again demonstrated that resolution was delayed (Krause et al 1978). Results were comparable whether patients still had their colons in situ $(\mathrm{n}=4)$ or had previously had a colectomy $(\mathrm{n}=6)$. In marked contrast, vascular responses were normal in those patients receiving 5-ASA (figure 1C,D).

In addition, during these studies, one UC patient that had been studied when off treatment presented with a flare of their colitis eight months later, and treatment was commenced with $4 \cdot 8 \mathrm{~g}$ Mesalazine. We retested this individual 10 months later whilst still on treatment, and found the previously heightened and protracted vascular response had normalized (figure 1D).

\section{Acute inflammatory reaction triggered by S. pneumoniae}

To establish whether the enhanced reactions were specific to Gram-negative organisms, we challenged four HC and three UC patients off 5-ASAs with S. pneumoniae. The response to this 
agent was less potent in both $\mathrm{HCs}$ and UC patients (figure 1E,G), but the exaggerated early response and delayed resolution were still evident in the UC patients $(\mathrm{p}=0 \cdot 02$; figure $1 \mathrm{~F}, \mathrm{G})$.

\section{Cellular inflammatory response}

To identify whether the exaggerated vascular responses were associated with differences in the populations of infiltrating cells, we sampled the acute inflammatory exudates by raising suction blisters over the inoculation sites at $4 \mathrm{~h}$ or $48 \mathrm{~h}$ after injection. The former captures initiation of acute inflammation where neutrophil ingress predominates (which is critical for clearance of the bacterial stimulus) (Smith et al., 2009); the latter represents a time at which resolution has occurred in $\mathrm{HC}$ but at which inflammation persists in UC . Early inflammatory reactions were hypercellular( $\mathrm{p}=0 \cdot 04$; figure $2 \mathrm{~A})$, due to an approximate doubling of neutrophil numbers $(\mathrm{p}=0 \cdot 05$; figure $2 \mathrm{~B})$; which was normalized in patients on treatment with 5ASA. Early mononuclear phagocyte influx was similar between the groups (figure 2C).

By 48h, the inflammatory infiltrate had largely cleared in HC (figure 2D), but exudates in UC patients (both off and on treatment) remained hypercellular $(\mathrm{p}=0 \cdot 007$ and $\mathrm{p}=0 \cdot 005$, respectively). In patients off treatment there were higher numbers of neutrophils $(p=0 \cdot 002$; figure 2E), and mononuclear phagocytes (figure $2 \mathrm{~F}$ ). Although the trend towards expansion of lymphocytes in UC $48 \mathrm{~h}$ blisters was not statistically significant $(\mathrm{p}=0 \cdot 08$; figure $2 \mathrm{G})$ there was a clear increase in $\mathrm{CD}^{+} \mathrm{T}$ cells $(\mathrm{p}=0 \cdot 001$; figure $2 \mathrm{H})$, with no differences in $\mathrm{CD}^{+}$(figure $\left.2 \mathrm{I}\right)$ or $\mathrm{B}$ cells. Additional phenotyping identified a $\mathrm{CD} 25^{\text {hi }} \mathrm{CD} 127^{\mathrm{lo}} \mathrm{CCR} 7^{-} \mathrm{CD} 45 \mathrm{RO}^{+}$dominant subset of the $\mathrm{CD}^{+} \mathrm{T}$ cells (supplementary figure 1A,B), which correspond to highly proliferative memory Tregs that preferentially localize to skin (Booth et al., 2010).

In UC-5ASA, at 48h there was an expansion of mononuclear phagocytes compared to $\mathrm{HC}$ $(\mathrm{p}=0 \cdot 03$; figure $2 \mathrm{~F})$, many of these cells exhibited a resolution macrophage phenotype 
(characterized by CD163 scavenger receptor expression; supplementary figure 1C) (Jenner et al., 2014). Neutrophils were also elevated ( $\mathrm{p}=0 \cdot 0009)$; however, in three patients off treatment and two UC-5ASA, a viability stain was included and demonstrated that, in UC at $48 \mathrm{~h}, 76 \cdot 2 \%$ of neutrophils were alive compared to only $18.8 \%$ in UC-5ASA (figure $2 \mathrm{E}$, inset). Corresponding values in $4 \mathrm{~h}$ neutrophils were $90.4 \%$ and $85 \cdot 3 \%$, respectively. In addition, $48 \mathrm{~h}$ neutrophil numbers correlated strongly with mononuclear phagocytes in HC and UC-5ASA $(\mathrm{r}=0 \cdot 610$, $\mathrm{p}=0 \cdot 03$ and $\mathrm{r}=0 \cdot 952, \mathrm{p}=0 \cdot 001)$, a characteristic associated with resolving acute inflammation but lost in UC on no therapy $(\mathrm{r}=0 \cdot 32, \mathrm{p}=0 \cdot 5)$.

\section{Cytokine secretion in vivo}

Next, we measured concentrations of 29 cytokines in blister exudates. Only interferon- $\gamma$ was significantly different at $4 \mathrm{~h}$, being higher in $\mathrm{HC}(\mathrm{p}=0 \cdot 04$; figure $3 \mathrm{~A})$. At $48 \mathrm{~h}$, macrophagederived chemokine (MDC), which contributes to Treg accumulation (Hao et al., 2016), was elevated in $\mathrm{HC}(\mathrm{p}=0.05$; figure $3 \mathrm{~B})$. After accounting for multiple testing, these findings argue against both the excess production of cytokines, or diminished scavenging of these mediators, as being responsible for the increased inflammation, unless non-classical mediators are involved.

\section{Lipid mediators in vivo}

As cytokine profiles did not explain cellular phenotypes, we assayed exudates for inflammatory lipid mediators, which are known to influence both initiation of acute inflammation and its resolution (Stables and Gilroy 2011)Multiple species were detected (see supplementary tables 2 and 3, and supplementary figure 2), of which in 4 h blisters only 13,14-dihydro-15-ketoprostaglandin $\mathrm{E}_{2}(\mathrm{p}=0 \cdot 01$; figure $3 \mathrm{C})$ and 13,14-dihydro-15-keto-prostaglandin $\mathrm{E}_{1}(\mathrm{p}=0 \cdot 02$; figure 3D) were significantly different, being elevated in UC and returning towards normal in 
UC-5ASA. These metabolites are the most stable products of PGE in vivo enzymatic degradation, and most reflective of overall production (Samuelsson and Green, 1974).

\section{Prostaglandin production in vitro}

Since $\mathrm{PGE}_{2}$ can be generated by neutrophils (Weissmann et al, 1982), we considered whether the observed increase might simply reflected elevated cell numbers. Consequently, we assayed $\mathrm{PGE}_{2}$ production following exposure to UVkEc in peripheral blood-derived leukocytes in vitro. We were unable to detect any generation by neutrophils from any subject, but when we examined macrophages, the pattern of secretion mirrored that observed in the in vivo exudate fluid with elevated levels of PGE2 in untreated UC ( $p=0 \cdot 004$; figure 3E) which reverted to normal in those patients treated with 5ASA.

\section{Role of OxoODE in resolving inflammation in UC-5ASA}

In UC-5ASA, lipid mediators in 4h blisters were comparable to HC, although 15 hydroxyeicosatrienoic acid (HETrE) was marginally lower ( $\mathrm{p}=0 \cdot 04$; figure $3 \mathrm{~F})$. In contrast, at 48h, their exudates contained higher concentrations of linoleic acid-derived hydroxy fatty acids 9-oxo-octadecadienoic acid (9-OxoODE; $\mathrm{p}=0 \cdot 05$; figure $3 \mathrm{G})$ and $13-\mathrm{OxoODE}(\mathrm{p}=0 \cdot 01$; figure 3H). As the hydroxyoctadecadienoic acid (HODE) precursors to these molecules are elevated during inflammation resolution (Tam et al., 2013), we assessed their effects on cytokine secretion by cultured macrophages, at concentrations comparable to those detected in vivo. Coincubation with UVkEc and increasing concentrations of 9-OxoODE led to dose-dependent suppression of TNF- $\alpha$ secretion ( $\mathrm{IC}_{50} \approx 100 \mathrm{nM}$; figure $3 \mathrm{I}$ ), with no impact on cell viability. Similar results were obtained with 13-OxoODE (supplementary figure 3), although this was less potent. As several oxidized fatty acids covalently bind and activate PPAR $\gamma$, we examined the impact of GW9662 a potent and selective PPAR $\gamma$ inhibitor(Seargent et al 2004) on TNF- $\alpha$ 
secretion. This agent completely abrogated the suppressive effects of 9-OxoODE on TNF- $\alpha$ secretion at concentrations of 10nM and 100nM (Figure 3I).

\section{Differential impact of 5ASA on HC}

Given the observed effects of 5ASA on inflammation in UC, we investigated the effect of this drug on the response of HCs that had been loaded with, and maintained on, slow-release oral mesalazine (HC-5ASA) to inoculation with UVkEC. We observed that treatment with this drug did not induce differences in cutaneous blood flow in the $5 \mathrm{HCs}$ (figure 4A), implying that rather than indiscriminately suppressing the general response, the effect of the 5ASAs in UC is more specifically directed to damping down the abnormal exuberant inflammation. Similarly, there were no differences in inflammatory cell infiltrates at $4 \mathrm{~h}$ (figure $4 \mathrm{~B}$ ), but $48 \mathrm{~h}$ reactions were hypercellular ( $\mathrm{p}=0.008$; figure $4 \mathrm{C}$ ), with $>90 \%$ of neutrophils found to be apoptotic. As in UC-5ASA, cytokines were unchanged at 4h (figure 4D), but by 48h (figure 4E) the levels of IL$13(\mathrm{p}=0 \cdot 03), \mathrm{TNF}-\alpha(\mathrm{p}=0 \cdot 03), \mathrm{MCP}-1(\mathrm{p}=0 \cdot 05), \mathrm{MCP}-4(\mathrm{p}=0 \cdot 003)$ and eotaxin-3 $(\mathrm{p}=0 \cdot 003)$ were minimally diminished. Measurements of the lipids showed that concentrations of 15HETrE were elevated ( $\mathrm{p}=0 \cdot 03$; figure 4F) and 19,20-dihydroxydocosapentaenoic acid diminished $(p=0 \cdot 03)$ at $4 \mathrm{~h}$; and at $48 \mathrm{~h}$ PGF2 $\alpha$ was reduced $(\mathrm{p}=0 \cdot 03$; figure $4 \mathrm{G})$.

\section{Discussion}

These studies demonstrate that the acute inflammatory response is increased in UC, with both an exaggerated onset, and delayed resolution This phenotype contrasts strongly with that observed in Crohn's disease, where acute inflammation is impaired, which results in delayed bacterial clearance and chronic granulomatous inflammation (Marks et al., 2006a; Smith et al., 2009, Segal AW 2019). This study demonstrates the hazards of conflating these two very different 
conditions together under the label of "Inflammatory Bowel Disease", particularly when investigating causal mechanisms with genetic screens such as GWAS(Huang eat al 2017). Excessive neutrophil recruitment and persistence at sites of bacterial inoculation recapitulate the pathological appearances of inflammation in the bowel in UC. The exaggerated reaction to $E$. coli could well help to explain the pathophysiology of UC. While the epithelium normally provides a highly efficient barrier to the bacteria laden faecal contents in health, insults such as infection or trauma allow luminal contents access to tissues of the bowel wall. Trauma could help to explain the observed distribution of UC, which extends proximally from the anus. Progressive dehydration of stool during transit through the colon increases the solidity of the faeces, and with that, the risk of frictional injury to the epithelial barrier. Alterations in genes that are important for maintaining the integrity of the mucus barrier and mucosa could compromise this barrier function and further increase exposure to enteric organisms (McGovern et al., 2015).

The observation of an exaggerated acute inflammatory response in the skin to both gutderived Gram-negative and to Gram-positive bacteria, raises the question as to why inflammation principally arises in the gastrointestinal tract in UC. This is probably related to the very large numbers of bacteria present in the bowel, readily available to enter the tissues if the mucosa is breeched. It also implies defects in more generic immune mechanisms, rather than specific pathogen-recognition receptors or antigen-directed lymphocyte responses. Similar results were previously reported following intradermal injection of killed Streptococcus pyogenes, which produced exaggerated pustular reactions in UC after 48h (Krause et al., 1978). This exaggerated inflammation may additionally provide causal mechanisms for the previously unexplained extraintestinal manifestations in UC (Greuter TI and Vavricka SR, 2019) to conditions affection the joints, lungs and skin such as pyoderma gangrenosum, bronchiectasis 
(Mateer et al., 2015), Sweet's syndrome (Ytting et al., 2005), and cutaneous infection with pustular eruptions (Hara et al., 2000).

The literature on $\mathrm{PGE}_{2}$ is divergent, with both pro-inflammatory and pro-resolution effects reported, and it may be regarded as a barometer of inflammation. The heightened production we describe here is consistent with previous observations of increased levels in blood and colonic mucosal macrophages and eosinophils in active and relapsing UC (Carty et al., 2000; Raab et al., 1995). The exogenous administration of $\mathrm{PGE}_{2}$ causes diarrhoea and pain, and may be a contributing factor to symptoms of the disease. More recently, phospholipase PLA2G2E, a primary regulator of arachidonic acid release for $\mathrm{PGE}_{2}$ generation which is upregulated by lipopolysaccharide (Murakami et al., 2002), has been consistently identified among GWAS UC susceptibility loci (McGovern et al., 2010; Silverberg et al., 2009).

5ASA drugs were originally developed without knowledge of their molecular target, and the pathways through which 5ASA exerts its influence on the clinical course of UC have been unclear. These drugs are structurally similar to aspirin and also inhibit cyclooxygenase. Our findings suggest that, by also promoting formation of 9-OxoODE and 13-OxoODE in UC, 5ASA exerts additional immunomodulatory effects through PPAR $\gamma$. This provides a mechanism for previous reports of loss of their ameliorating effects in murine colitis using PPAR $\gamma$ heterozygous knockout animals (Rousseaux et al., 2005); antineoplastic effects in colorectal cancer cells lines associated with PPAR $\gamma$-related transcriptional activity (Schwab et al., 2008) and abolition of these in vivo by GW9662 (Rousseaux et al., 2013); and preliminary reports of efficacy of PPAR $\gamma$ agonists in murine and human colitis (Celinski et al., 2013; Lewis et al., 2008). We postulate that divergent effects in HC-5ASA likely reflect lower precursor substrate release from cell 
membranes, resulting in decreased shunting towards hydroxy-octadecanoic acid (HODE) (Murakami et al., 2010).

The principal limitation of this study is that bacterial challenge had to be conducted in the skin, rather than in the gastrointestinal tract. This was considered necessary given the restrictive practicalities of injecting bacteria then sampling in the bowel, as well as potential safety implications of provoking excessive neutrophil recruitment in a location where breaching the mucosal barrier could lead to ongoing exposure and propagated responses (particularly given an extreme reaction seen previously in a UC patient) (Marks et al., 2006b). Likewise, as the experiments required the collection of cells trafficking to inflammatory sites, it would be impractical to recover these cells from specific sites in the bowel.

Several of these findings are immediately amenable to clinical translation. The observations that inflammatory lipid mediators play central roles in the pathogenesis of UC and in the therapeutic effects of 5ASA, the major therapeutic tool in UC, are important for the understanding of this disease and for the development of more effective therapy. The acute inflammatory phenotype identifies new biomarkers that should be evaluated for determination of therapeutic efficacy of established and novel medications. Existing PPAR $\gamma$ agonists can be revisited as therapeutics, while more gut-specific molecules are developed alongside stable 9OxoODE and 13-OxoODE analogues; these would be predicted to enhance inflammation resolution without increasing susceptibility to infection. Likewise, the potential for bacterial challenge as a diagnostic test in patients with indeterminate inflammatory bowel disease might be useful in distinguishing between UC and Crohn's disease. 


\section{Materials and methods}

\section{Patients with ulcerative colitis and healthy control participants}

Patients with endoscopically and histologically proven UC were identified through the gastroenterology clinics at University College London Hospitals (UCLH). Healthy controls (HC) were identified through the Division of Medicine, University College London (UCL), and were defined as individuals with no history of inflammatory bowel disease or other active inflammation disorder. All patients had quiescent disease, defined as a partial Mayo score of 0 and (where available) no active inflammation on recent endoscopy and serum C-reactive protein $\leq 5 \mathrm{mg} / \mathrm{L}$, but previous left-sided or extensive colitis. Each patient who fulfilled the eligibility criteria was approached regarding their interest in participating in research, with a median time from first screening to participation of 35 days. Partial Mayo scores of 0 were re-confirmed on the day of bacterial injection. No participant had received corticosteroid, immunosuppressant or anti-TNF drug therapy within three months, or non-steroidal anti-inflammatory drugs within one week of participation. For UC-5ASA patients, 5ASA was taken orally, and consisted of one of mesalazine $1.2-4.8 \mathrm{~g}$ daily $(\mathrm{n}=8)$, balsalazide $750 \mathrm{mg}-3 \mathrm{~g}$ daily $(\mathrm{n}=2)$ or sulfasalazine $2 \mathrm{~g}$ daily $(\mathrm{n}=1)$. Where 5ASA was given to HC, this was provided as Mezavant XL $4 \cdot 8 \mathrm{~g}$ orally, taken once a day for five days starting 48 hours prior to bacterial injection. Exclusion criteria included age $<18$ years, HIV infection, pregnancy, breastfeeding, or personal or family history of pyoderma gangrenosum. No participants had active infection while participating in the study. Studies were approved by the National Research Ethics Service Committees London Harrow (project number 13/LO/1541) and Surrey Borders (project number 10/H0806/115). Written informed consent was obtained from all participants.

\section{Preparation of bacteria for injection into human participants}


Antibiotic-sensitive clinical isolates of E. coli strain NCTC 10418 (Public Health England, UK) and S. pneumoniae serotype 4 (TIGR4 strain; gift from JS Brown) were used for bacterial injection studies. E. coli were grown overnight in Luria Broth (Sigma-Aldrich, MO, USA), and S. pneumoniae in Colombia blood agar (E\&O Laboratories, UK) followed by Todd-Hewitt broth plus $0.5 \%$ yeast extract (Sigma), both at $37^{\circ} \mathrm{C}$ in $5 \% \mathrm{CO}_{2}$. Bacteria were washed twice in sterile phosphate-buffered saline (PBS; 2,500g, 20min, $4^{\circ} \mathrm{C}$ ), then plated in a sterile petri dish. Counts were determined by optical density for $E$. coli $\left(\mathrm{OD}_{600}=0 \cdot 365=10^{8}\right.$ organisms $\left./ \mathrm{ml}\right)$ (Smith et al., 2009), and direct counting through serial dilution and plating for S. pneumoniae. Bacteria were killed by exposure to a 302nm UV light source (ChemiDoc; Bio-Rad, UK) for 60 min, washed and resuspended in sterile $0.9 \%$ saline at a concentration of $1 \cdot 5 \times 10^{8} / \mathrm{ml}$. Samples were aliquoted into sterile Eppendorf tubes and frozen at $-80^{\circ} \mathrm{C}$ until use. Non-viability was confirmed in multiple cultures by the UCLH Microbiology Department. Either $1 \cdot 5 \times 10^{7}$ E. coli or $7 \cdot 5 \times 10^{7} \mathrm{~S}$. pneumoniae were injected into the volar aspect of each forearm. Bacterial numbers for inoculation were determined by dose-response studies in $\mathrm{HC}$, including a safety margin given a previous reaction in UC (Marks et al., 2006b). Blood flow at these sites was subsequently quantified using laser Doppler (Moor LDI-HIR, Moor Instruments Ltd, UK), analysed using Moor LDI software (Version 5), and calculated as the product of mean signal and area.

\section{Suction blisters}

To sample exudates, a 10mm suction blister was raised over inoculation sites, $4 \mathrm{~h}$ or $48 \mathrm{~h}$ following injection. A blister chamber connected to negative pressure suction (NP-4, Electronic Diversities Ltd, MD, USA) was securely fastened to the forearm. Negative pressure was applied (starting at $2 \mathrm{inHg}$ and escalating at $10 \mathrm{~min}$ intervals to $5-12 \mathrm{inHg}$ ) until the epidermis separated, forming a blister covering the surface area of the aperture. Pressure was then reduced to baseline 
at 1 inHg every $5 \mathrm{~min}$ and the chamber removed. Blisters were pierced on their lateral border using a $26 \cdot 5 \mathrm{G}$ needle and exudates collected into tubes containing $50 \mu 13 \%$ sodium citrate (Sigma) in PBS (Gibco, UK). The blister area was cleaned using 0.5\% Cetrimide spray and dressed. Collection tubes were weighed to calculate blister volume.

\section{Analysis of cells in blister fluid}

Exudates were centrifuged at $1,000 \mathrm{~g}$ for $5 \mathrm{~min}$ at $10^{\circ} \mathrm{C}$, after which supernatants were aliquoted and immediately frozen at $-80^{\circ} \mathrm{C}$. Cell pellets were resuspended in $100 \mu \mathrm{l}$ ACK erythrocyte lysis buffer (Lonza, Switzerland) for $1 \mathrm{~min}$, then centrifuged at $1,000 \mathrm{~g}$ for $5 \mathrm{~min}$ at $10^{\circ} \mathrm{C}$. Supernatants were discarded and cells resuspended in $100 \mu 1$ PBS with $5 \%$ foetal bovine serum (FBS; Gibco, UK) and $0 \cdot 1 \%$ sodium azide (Sigma). Cell were counted with a manual haemocytometer. Leukocyte subpopulations in blisters and peripheral blood samples were characterized by polychromatic flow cytometry, using a previously described gating strategy (Motwani et al., 2016). For isolation of circulating leukocytes, $4 \mathrm{ml}$ blood was collected, erythrocytes lysed with ACK buffer, and cells washed with PBS prior to resuspension in PBS/5\% FBS. Cells were incubated with an antibody cocktail (supplementary table 1) for 30min at room temperature in the dark. Stained samples were washed in PBS containing 1\% FBS and 2mM EDTA (Sigma, UK), then centrifuged at $800 \mathrm{rpm}$ for $5 \mathrm{~min}$ at $4^{\circ} \mathrm{C}$. Cells were resuspended in PBS/5\%FBS then fixed in an equal volume of $1 \%$ paraformaldehyde and stored in the dark at $4^{\circ} \mathrm{C}$ prior to analysis on a BD LSR Fortessa ${ }^{\mathrm{TM}}$ or LSR2 flow cytometer (BD Biosciences, UK). Data were analysed using FlowJo software (FlowJo LLC, OR, USA). Clearance of cells between two time points was defined as $\left(\mathrm{n}_{1}-\mathrm{n}_{2}\right) / \mathrm{t}$, where $\mathrm{n}$ is the number of cells present at a specified time point, and the interval between time points.

\section{Analysis of cytokines and lipid mediators in blister fluid}


Blister supernatants were assayed for cytokines using the V-PLEX Human Cytokine 30-Plex Kit (Meso Scale Delivery, MD, USA). Lipid mediators were analysed by ultraperformance liquid chromatography coupled to electrospray ionization tandem mass spectrometry (UPLC/ESIMS/MS), based on protocols published previously.(Kendall et al., 2015; Massey and Nicolaou, 2013) Samples (30-120 $\mu 1)$ were defrosted on ice and adjusted to $15 \%$ (v/v) methanol:water. Internal standards (20ng each of PGB $_{2}-d 4$, 12-HETE- $d 8,8$,9-DHET- $d 11$ and 8(9)EET- $d 11$ ) were added to each sample and incubated on ice for $30 \mathrm{~min}$. $\mathrm{pH}$ was adjusted to $3 \cdot 0$ with $1 \mathrm{M} \mathrm{HCl}$. Acidified samples were immediately applied to preconditioned solid-phase cartridges (C18-E; Phenomenex, Macclesfield, UK), washed with various solvents, and lipid mediators eluted with methyl formate (Massey and Nicolaou, 2013). UPLC/ESI-MS/MS analysis was performed on a UPLC pump (Acquity, Waters, Wilmslow, UK) coupled to an electrospray ionization triple quadruple mass spectrometer (Xevo TQ-S, Waters, Wilmslow, UK). Chromatographic separation was performed on a C18 column (Acquity UPLC BEH, $1 \cdot 7 \mu \mathrm{m}, 2 \cdot 1 \times 50 \mathrm{~mm}$; Waters, Wilmslow, UK). Quantitative analysis was based on multiple reaction monitoring-based assays as previously reported (Kendall et al., 2015; Massey and Nicolaou, 2013). Calibration lines were constructed using commercially available standards (Cayman Chemicals, MI, USA).

\section{Cytokine secretion by neutrophils and macrophages in vitro}

Peripheral blood neutrophils were isolated by mixing $20 \mathrm{ml}$ venous blood with $10 \%$ dextran (MP Biomedicals, OH, USA) to a final concentration of $1 \%$. This was sedimented for $1 \mathrm{~h}$ then the top layer centrifuged over Lymphoprep (Alere Technologies, Norway) at 2,000rpm for 10min at $20^{\circ} \mathrm{C}$. Supernatant was removed and erythrocytes lysed with $\mathrm{ddH}_{2} \mathrm{O}$. An equal volume of $1 \cdot 8 \%$ saline (Sigma) was added to restore normal tonicity, then cells centrifuged at $1,500 \mathrm{rpm}$ for $5 \mathrm{~min}$ 
at $20^{\circ} \mathrm{C}$. Cells were washed twice in PBS and resuspended in X-Vivo medium (Lonza, Switzerland) at $10^{6}$ cells $/ \mathrm{ml}$ before re-plating in tissue culture 96 -well plates.

To create macrophage cultures, peripheral blood mononuclear cells were isolated by centrifugation over Lymphoprep at $2,000 \mathrm{rpm}$ for $30 \mathrm{~min}$ at $20^{\circ} \mathrm{C}$, then washed three times with phosphate-buffered saline. Cells were resuspended in RPMI-1640 GlutaMAX supplement medium (Gibco, UK) containing 100U/ml penicillin/streptomycin (Gibco, UK) and plated at $10^{7}$ cells/ml in tissue culture dishes (Nunc, Denmark). After 90min, medium was exchanged with RPMI-1640 containing 10\% FBS, 20mM HEPES (Sigma) and 100U/ml penicillin/streptomycin. These were cultured for 5 days at $37^{\circ} \mathrm{C}, 5 \% \mathrm{CO}_{2}$, with medium supplemented on day 2 . On day 5, macrophages were washed three times in PBS, scraped, resuspended at $10^{6} / \mathrm{ml}$ in serum-free X-Vivo medium, and re-plated in 96-well tissue culture plates (Falcon, USA). Cells were allowed to adhere overnight prior to stimulation.

Cultured cells were incubated with or without $10^{5} \mathrm{UV}$-killed E. coli. In some experiments, macrophages were co-incubated with 9-OxoODE, 13-OxoODE, GW9662 (Cayman Chemical) or vehicle controls. $\mathrm{PGE}_{2}$ and TNF- $\alpha$ secretion were assessed in culture supernatants by ELISA (R\&D Systems, UK). Numbers of viable cells in each well were ascertained using Cell Counting Kit-8 (Sigma-Aldrich). All assays utilised protocols from the manufacturers.

\section{Statistical analysis}

Statistical tests were performed using Graphpad Prism version 7·0 (GraphPad Software, CA, USA). Data are presented as mean \pm sem unless otherwise stated. Based on observations in previous studies, a sample size of 10 participants per group would be sufficient to detect clinically significant differences in vascular responses with power $>95 \%(\alpha=0 \cdot 05, \beta=0 \cdot 5)($ Marks et al., 2006b; Rahman et al., 2010). The Mann-Whitney U test was used for single comparisons, 
and Kruskal-Wallis ANOVA with Dunn post-tests or two-way ANOVA for multiple comparisons. Where parametric analyses were used, samples were assessed for normality using the D’Agostino and Pearson normality test. Significance values refer to comparison with HC under the same conditions unless otherwise stated. $\mathrm{P}$ values $<0 \cdot 05$ were considered significant.

During the study, one blister (48h collection in a UC-5ASA patient) ruptured during application of negative pressure, and therefore data on cells and soluble mediators at this time point from this individual were missing; however, all other measurements from this patient, and all samples from other participants, were included in the analyses.

\section{Role of the funding source}

The study sponsor had no role in study design; data collection, interpretation or analysis; or writing of the report.

\section{Contributions}

DJBM, AN, DWG and AWS were responsible for the study design. DJBM, RV, FZR, SM and SLB provided clinical care of the patients, and provided clinical phenotyping. DJBM undertook all experiments in conjunction with other authors as follows: RW performed flow cytometry, multiplex cytokine arrays, cell fractionation and culture, cell stimulation and inhibitor studies, and ELISA; ACK performed mass spectrometry for lipid analysis; and MM assisted in development of the blister model. AN planned the lipidomics study, designed the experiments and was responsible for interpretation of lipidomic data. DJBM drafted the initial manuscript, and all authors participated in critical revision. All authors approved the completed article. 


\section{Declaration of interests}

DJBM is currently an employee of, and has share/stock options with, GSK, but had no affiliation with GSK at the time these studies were conducted. GSK has had no role in the conduct or interpretation of, or decision to publish, this report.

\section{Acknowledgments}

This work was funded by the Wellcome Trust, project reference 100098/Z/12/Z. We gratefully acknowledge Prof JS Brown for provision of the streptococcus strains, and M Bonaiti for microbiology input. 


\section{References}

Booth, N.J., A.J. McQuaid, T. Sobande, S. Kissane, E. Agius, S.E. Jackson, M. Salmon, F. Falciani, K. Yong, M.H. Rustin, A.N. Akbar, and M. Vukmanovic-Stejic. 2010. Different proliferative potential and migratory characteristics of human CD4+ regulatory T cells that express either CD45RA or CD45RO. J Immunol 184:4317-4326.

Carty, E., M. De Brabander, R.M. Feakins, and D.S. Rampton. 2000. Measurement of in vivo rectal mucosal cytokine and eicosanoid production in ulcerative colitis using filter paper. Gut 46:487-492.

Celinski, K., T. Dworzanski, R. Fornal, A. Korolczuk, A. Madro, T. Brzozowski, and M. Slomka. 2013. Comparison of anti-inflammatory properties of peroxisome proliferator-activated receptor gamma agonists rosiglitazone and troglitazone in prophylactic treatment of experimental colitis. J Physiol Pharmacol 64:587-595.

de Souza, H.S., and C. Fiocchi. 2016. Immunopathogenesis of IBD: current state of the art. Nat Rev Gastroenterol Hepatol 13:13-27.

Eaden JA, Abrams KR and Mayberry JF. 2001. The risk of colorectal cancer in ulcerative colitis: a meta-analysis. Gut 48:526-35.

Gilroy, D., and R. De Maeyer. 2015. New insights into the resolution of inflammation. Semin Immunol 27:161-168.

Greuter T1 and Vavricka SR. 2019. Extraintestinal manifestations in inflammatory bowel disease - epidemiology, genetics, and pathogenesis.Expert Rev Gastroenterol Hepatol. 13:307-317.

Hao, S., X. Han, D. Wang, Y. Yang, Q. Li, X. Li, and C.H. Qiu. 2016. Critical role of CCL22/CCR4 axis in the maintenance of immune homeostasis during apoptotic cell clearance by splenic CD8alpha(+) CD103(+) dendritic cells. Immunology 148:174-186.

Hara, H., Wakui, F., Fujitsuka, A., Ochiai, T., and T. Morishima. 2000. Subcutaneous abscesses in a patient with ulcerative colitis. J Am Acad Dermatol 42:363-365.

Huang H, Fang M, Jostins L, Umicevic-Mirkov M, Boucher G, Anderson CA, et al. 2017. Fine-mapping inflammatory bowel disease loci to single-variant resolution. Nature . 547:173-178.

Jenner, W., M. Motwani, K. Veighey, J. Newson, T. Audzevich, A. Nicolaou, S. Murphy, R. Macallister, and D.W. Gilroy. 2014. Characterisation of leukocytes in a human skin blister model of acute inflammation and resolution. PLoS One 9:e89375.

Kendall, A.C., S.M. Pilkington, K.A. Massey, G. Sassano, L.E. Rhodes, and A. Nicolaou. 2015. Distribution of bioactive lipid mediators in human skin. J Invest Dermatol 135:1510-1520.

Krause, U., G. Michaelsson, and L. Juhlin. 1978. Skin reactivity and phagocytic function of neutrophil leucocytes in Crohn's disease and ulcerative colitis. Scand J Gastroenterol 13:71-75.

Lewis, J.D., G.R. Lichtenstein, J.J. Deren, B.E. Sands, S.B. Hanauer, J.A. Katz, B. Lashner, D.H. Present, S. Chuai, J.H. Ellenberg, L. Nessel, G.D. Wu, and G. Rosiglitazone for Ulcerative Colitis Study. 2008. Rosiglitazone for active ulcerative colitis: a randomized placebo-controlled trial. Gastroenterology 134:688-695.

Marks, D.J., M.W. Harbord, R. MacAllister, F.Z. Rahman, J. Young, B. Al-Lazikani, W. Lees, M. Novelli, S. Bloom, and A.W. Segal. 2006a. Defective acute inflammation in Crohn's disease: a clinical investigation. Lancet 367:668-678.

Marks, D.J., F.Z. Rahman, M. Novelli, R.C. Yu, S. McCartney, S. Bloom, and A.W. Segal. 2006b. An exuberant inflammatory response to E coli: implications for the pathogenesis of ulcerative colitis and pyoderma gangrenosum. Gut 55:1662-1663.

Massey, K.A., and A. Nicolaou. 2013. Lipidomics of oxidized polyunsaturated fatty acids. Free Radic Biol Med 59:45-55.

Mateer, S.W., S. Maltby, E. Marks, P.S. Foster, J.C. Horvat, P.M. Hansbro, and S. Keely. 2015. Potential mechanisms regulating pulmonary pathology in inflammatory bowel disease. J Leukoc Biol 98:727-737.

McGovern, D.P., A. Gardet, L. Torkvist, P. Goyette, et al 2010. Genome-wide association identifies multiple ulcerative colitis susceptibility loci. Nat Genet 42:332-337.

McGovern, D.P., S. Kugathasan, and J.H. Cho. 2015. Genetics of Inflammatory Bowel Diseases. Gastroenterology 149:1163-1176 e1162.

Molodecky, N.A., I.S. Soon, D.M. Rabi, W.A. Ghali, M. Ferris, G. Chernoff, E.I. Benchimol, R. Panaccione, S. Ghosh, H.W. Barkema, and G.G. Kaplan. 2012. Increasing incidence and prevalence of the inflammatory bowel diseases with time, based on systematic review. Gastroenterology 142:46-54 e42; quiz e30. 
Motwani, M.P., J.D. Flint, R.P. De Maeyer, J.N. Fullerton, A.M. Smith, D.J. Marks, and D.W. Gilroy. 2016. Novel translational model of resolving inflammation triggered by UV-killed E. coli. J Pathol Clin Res 2:154-165.

Murakami, M., Y. Taketomi, C. Girard, K. Yamamoto, and G. Lambeau. 2010. Emerging roles of secreted phospholipase A2 enzymes: Lessons from transgenic and knockout mice. Biochimie 92:561-582.

Murakami, M., K. Yoshihara, S. Shimbara, G. Lambeau, A. Singer, M.H. Gelb, M. Sawada, N. Inagaki, H. Nagai, and I. Kudo. 2002. Arachidonate release and eicosanoid generation by group IIE phospholipase A(2). Biochem Biophys Res Commun 292:689-696.

Raab, Y., C. Sundberg, R. Hallgren, L. Knutson, and B. Gerdin. 1995. Mucosal synthesis and release of prostaglandin E2 from activated eosinophils and macrophages in ulcerative colitis. Am J Gastroenterol 90:614-620.

Rahman, F.Z., A.M. Smith, B. Hayee, D.J. Marks, S.L. Bloom, and A.W. Segal. 2010. Delayed resolution of acute inflammation in ulcerative colitis is associated with elevated cytokine release downstream of TLR4. PLoS One 5:e9891.

Rousseaux, C., N. El-Jamal, M. Fumery, C. Dubuquoy, O. Romano, D. Chatelain, A. Langlois, B. Bertin, D. Buob, J.F. Colombel, A. Cortot, P. Desreumaux, and L. Dubuquoy. 2013. The 5-aminosalicylic acid antineoplastic effect in the intestine is mediated by PPARgamma. Carcinogenesis 34:2580-2586.

Rousseaux, C., B. Lefebvre, L. Dubuquoy, P. Lefebvre, O. Romano, J. Auwerx, D. Metzger, W. Wahli, B. Desvergne, G.C. Naccari, P. Chavatte, A. Farce, P. Bulois, A. Cortot, J.F. Colombel, and P. Desreumaux. 2005. Intestinal antiinflammatory effect of 5-aminosalicylic acid is dependent on peroxisome proliferatoractivated receptor-gamma. J Exp Med 201:1205-1215.

Samuelsson, B., and K. Green. 1974. Endogenous levels of 15-keto-dihydro-prostaglandins in human plasma. Parameters for monitoring prostaglandin synthesis. Biochem Med 11:298-303.

Schwab, M., V. Reynders, S. Loitsch, Y.M. Shastri, D. Steinhilber, O. Schroder, and J. Stein. 2008. PPARgamma is involved in mesalazine-mediated induction of apoptosis and inhibition of cell growth in colon cancer cells. Carcinogenesis 29:1407-1414.

Seargent JM, Yates EA and Gill JH. 2004. GW9662, a potent antagonist of PPARgamma, inhibits growth of breast tumour cells and promotes the anticancer effects of the PPARgamma agonist rosiglitazone, independently of PPARgamma activation. Br J Pharmacol;143:933-7.

Silverberg, M.S., J.H. Cho, J.D. Rioux, D.P. McGovern, et al 2009. Ulcerative colitis-risk loci on chromosomes 1 p36 and 12q15 found by genome-wide association study. Nat Genet 41:216-220.

Segal, AW 2019. Studies on patients establish Crohn's disease as a manifestation of impaired innate immunity. J Intern Med. 2019 May 28. doi: 10.1111/joim.12945.

Smith, A.M., F.Z. Rahman, B. Hayee, S.J. Graham, D.J. Marks, et al. 2009. Disordered macrophage cytokine secretion underlies impaired acute inflammation and bacterial clearance in Crohn's disease. J Exp Med 206:1883-1897.

Stables MJ1, Gilroy DW 2011.Old and new generation lipid mediators in acute inflammation and resolution. Prog Lipid Res. 50:35-51.

Tam, V.C., O. Quehenberger, C.M. Oshansky, R. Suen, A.M. Armando, P.M. Treuting, P.G. Thomas, E.A. Dennis, and A. Aderem. 2013. Lipidomic profiling of influenza infection identifies mediators that induce and resolve inflammation. Cell 154:213-227.

Ungaro, R., S. Mehandru, P.B. Allen, L. Peyrin-Biroulet, and J.F. Colombel. 2017. Ulcerative colitis. Lancet 389:1756-1770.

Verstockt, B., M. Ferrante, S. Vermeire, and G. Van Assche. 2018. New treatment options for inflammatory bowel diseases. J Gastroenterol 53:585-590.

Weissmann G, Serhan C, Korchak HM and Smolen JE. 1982. Neutrophils: release of mediators of inflammation with special reference to rheumatoid arthritis. Ann N Y Acad Sci;389:11-24.

Ytting, H., Vind, I., Bang, D., and P. Munkholm. 2005. Sweet's syndrome--an extraintestinal manifestation in inflammatory bowel disease. Digestion 72:195-200. 


\section{Figure legends}

Figure 1: Acute inflammatory responses induced by intradermal inoculation of killed bacteria

(A) Local vascular responses provoked by E. coli were observed in $\mathrm{HC}(\mathrm{n}=13)$ within $4 \mathrm{~h}$, peaked by $24 \mathrm{~h}$ and almost completely resolved within $48 \mathrm{~h}$. (B) Responses in UC $(n=10)$ were of greater magnitude, and slower to resolve, except in (C) UC-5ASA $(n=11)$ treatment, in whom blood flow normalised. (D) Blood flow responses to UVkEC were consistently more pronounced and slower to resolve in UC than in $\mathrm{HC}$ or UC-5ASA (resolution index, calculated as rate of change of blood flow between $24 \mathrm{~h}$ and $48 \mathrm{~h}$, inset and confirms impaired resolution). One UC patient participated twice, initially off treatment (points $A_{1}$ and $A_{2}$ ) and subsequently while taking 5ASA $\left(\mathrm{B}_{1}\right.$ and $\mathrm{B}_{2}$ ). (E) Blood flow responses induced by $S$. pneumoniae followed a similar time course but were less extensive in $\mathrm{HC}(\mathrm{n}=4)$, but $(\mathrm{F}) \mathrm{UC}(\mathrm{n}=3)$ the enhanced peaks and delayed resolution followed the same pattern. (G) Aggregate responses to UV-killed S. pneumoniae confirmed that the abnormality in UC applied to Gram-positive as well as Gram-negative bacteria; at $48 \mathrm{~h}$, reactions in UC patients were slowly resolving in two patients but continued to progress in one individual. Data shown as mean \pm sem. 
Figure 2: Cellular response to $U \mathrm{VkEc}$

(A) By 4h, UC blisters ( $n=7)$ contained approximately double the number of cells compared to HC $(n=13)$; this was normalised in UC-5ASA $(n=9)$. The increased cell numbers were due to expansion of the (B) neutrophil population, whereas (C) the numbers of mononuclear phagocytes were unchanged. (D) In 48h blisters, cells had almost completely cleared in HC, whereas there were excess numbers in both UC groups. (E) There were high numbers of neutrophils in both UC and UC-5ASA participants, but inclusion of a live-dead stain (inset) revealed that in $4 \mathrm{~h}$ blisters (green) most of these cells in UC (n=3) were alive (1), but by $48 \mathrm{~h}$ the majority in UC-5ASA $(n=2)$ were non-viable (d). At 48h, (F) mononuclear phagocyte numbers were increased in both UC groups, and $(\mathrm{G})$ there was a trend towards higher lymphocyte numbers in UC patients off treatment; this was due to expansion of (H) CD4 but not (I) CD8 T cells. Data shown as mean \pm sem.

\section{Figure 3: Inflammatory mediators triggered by UVkEc}

Cytokine concentrations in (A) 4h and (B) 48h blisters, assayed from 13 HC, 7 UC and 9 UC5ASA. Lipidomic analysis revealed increased concentrations of (C) $\mathrm{PGE}_{2}$ and (D) $\mathrm{PGE}_{1}$ metabolites in $4 \mathrm{~h}$ blisters in UC. (E) In separate experiments, this was recapitulated in vitro in peripheral blood monocyte-derived macrophages stimulated with UVkEC from $5 \mathrm{HC}, 6 \mathrm{UC}$ and 13 UC-5ASA. In UC-5ASA, (F) HETrE was also reduced in 4h blisters, whereas production of (G) 9-OxoODE and (H) 13-OxoODE was enhanced in 48h blisters. (I) 9-OxoODE caused a dose-dependent suppression of TNF- $\alpha$ secretion from E. coli (EC)-stimulated macrophages in vitro ( $\mathrm{n}=3$ per condition). This was fully reversed by co-incubation with the PPAR $\gamma$ antagonist GW9662. Data shown as mean \pm sem. 
Figure 4: Effects of 5ASA treatment on the acute inflammatory response to $\mathrm{UVkEC}$ in $\mathrm{HC}$

(A) Blood flow was unchanged in HC-5ASA subjects $(n=5)$ compared with HC on no treatment $(n=5)$. Blister cellular profiles were no different at (B) 4h, but at (C) $48 \mathrm{~h}$ all principal cell types were present at greater numbers. Blister cytokine profiles at (D) 4 h were unchanged, but (E) subtle variations were seen at $48 \mathrm{~h}$. Alterations were also observed in the concentrations of $(\mathrm{F})$ 15-HETrE at 4h, and (G) PGF2 $\alpha$ at $48 \mathrm{~h}$. Data shown as mean \pm sem.

\section{Supplementary Materials}

\section{Supplementary Figure 1: Additional cell phenotyping in blister exudates}

(A) Among CD4 lymphocytes in UC patient blood and blisters, a population of CD25 ${ }^{\text {hi }}$ CD127 were identified that correspond to a known Treg phenotype; (B) these contributed to a substantial proportion of T cells within UC blisters. (C) In $48 \mathrm{~h}$ blisters, the proportion of resolution macrophages (as identified by up-regulation of the scavenger receptor CD163) was increased in UC-5ASA. $* \mathrm{p}<0 \cdot 05, * * \mathrm{p}<0 \cdot 01, * * * \mathrm{p}<0 \cdot 001$

\section{Supplementary Figure 2: Absence of detectable resolvins in blister fluid}

Chromatograms showing an absence of detectable resolvins $\mathrm{RvE}_{1}, \mathrm{RvD}_{1}$ and $\mathrm{RvD}_{2}$ in a representative blister fluid sample. Resolvin standards are shown at 30pg on-the-column and 2pg on-the-column, but were undetectable in all samples. The limits of detection for $\mathrm{RvE}_{1}, \mathrm{RvD}_{1}$ and $\mathrm{RvD}_{2}$ have been calculated as $180 \mathrm{fg} / \mu 1,50 \mathrm{fg} / \mu 1$ and $90 \mathrm{fg} / \mu \mathrm{l}$ blister fluid, respectively. 


\section{Supplementary Figure 3: Effect of 13-OxoODE on macrophage TNF- $\alpha$ secretion}

13-OxoODE caused a dose-dependent suppression of TNF- $\alpha$ secretion from E. coli (EC)-

stimulated macrophages in vitro. This was fully reversed by co-incubation with the PPAR $\gamma$ antagonist GW9662. **p=0·01.

Supplementary Table 1. Antibody panels used in flow cytometry experiments

Supplementary Table 2. Lipid mediators in 4 h blisters Concentrations in pg/ml (mean \pm sem).

Supplementary Table 3. Lipid mediators in 48 h blisters Concentrations in pg/ml (mean \pm sem). 
Figure 1
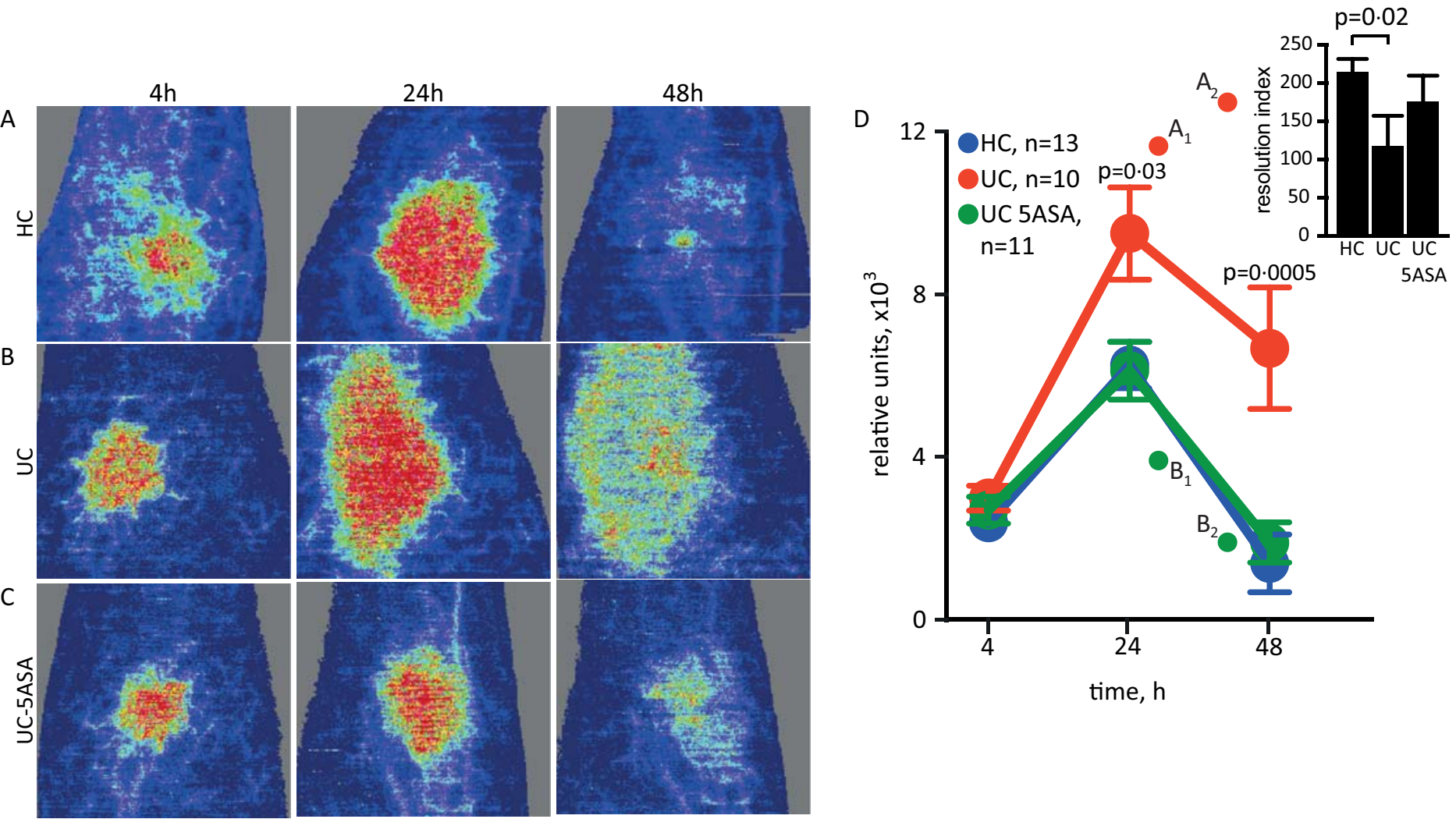

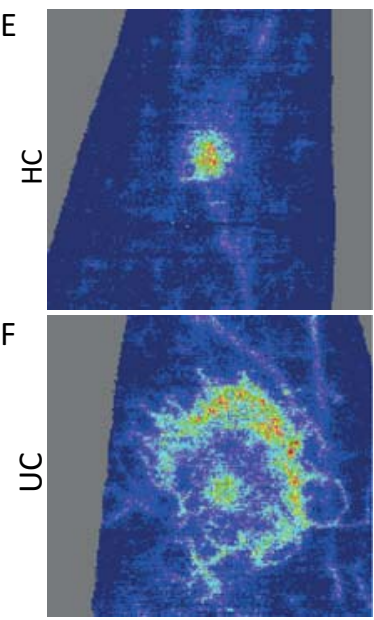

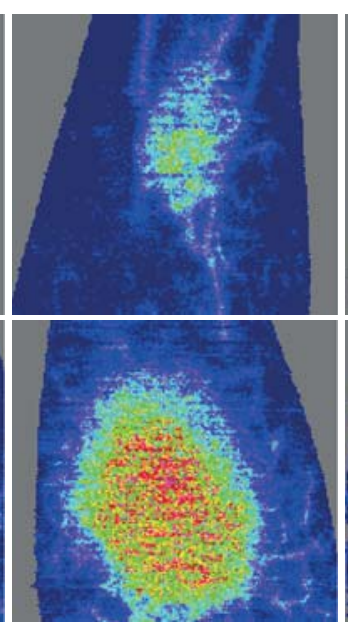

$500 \quad 1,000$

perfusion units

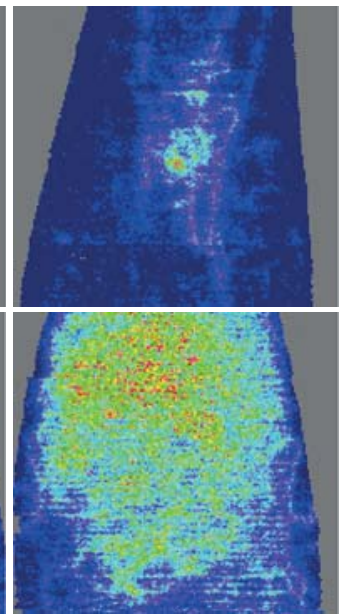

G

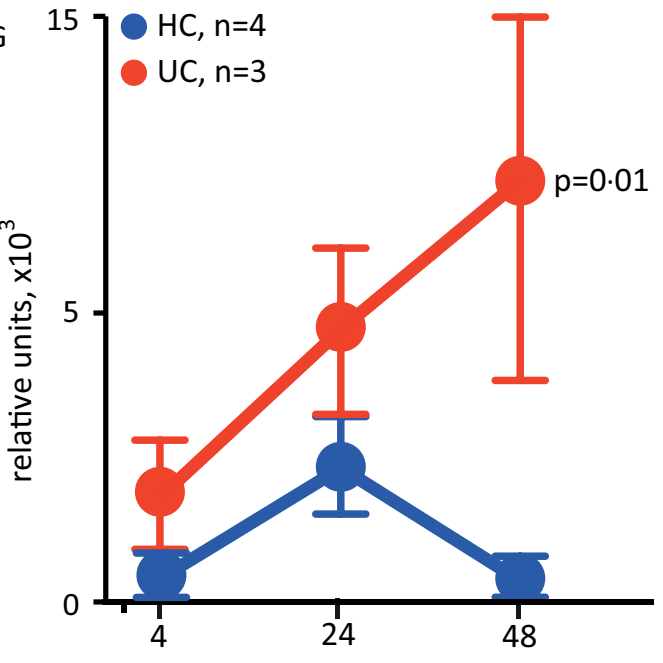

time, $\mathrm{h}$ 
Figure 2

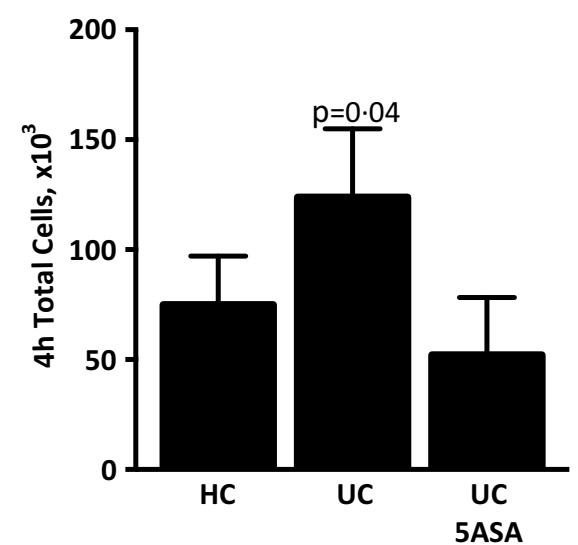

B

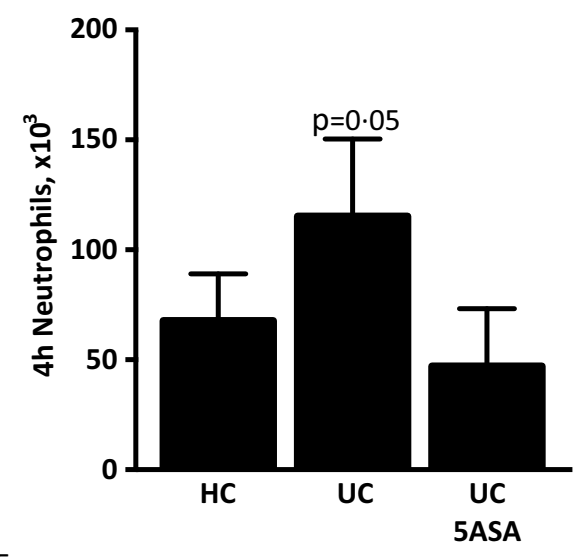

E

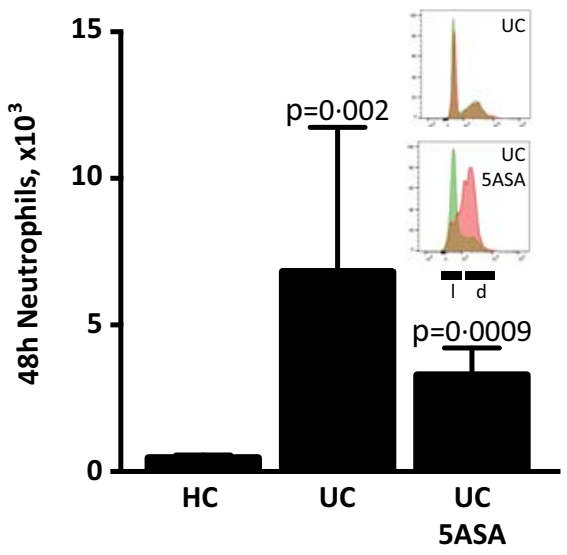

H

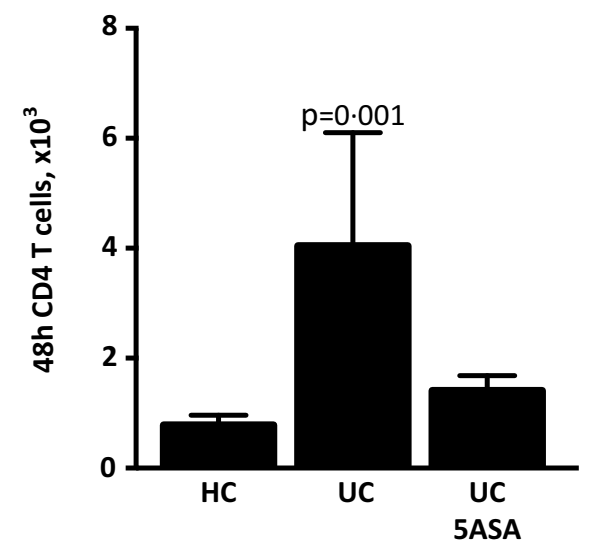

C

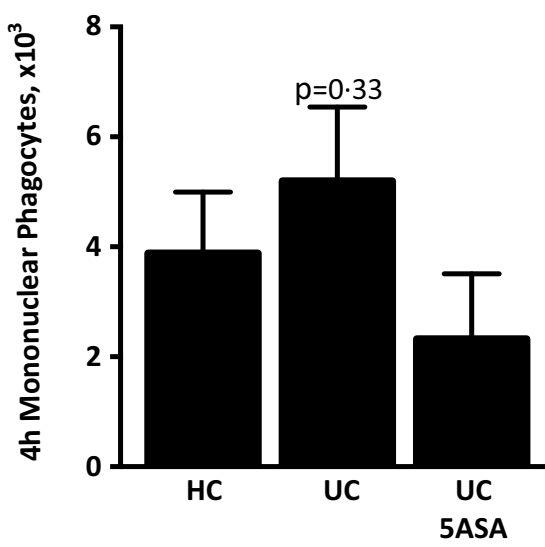

F
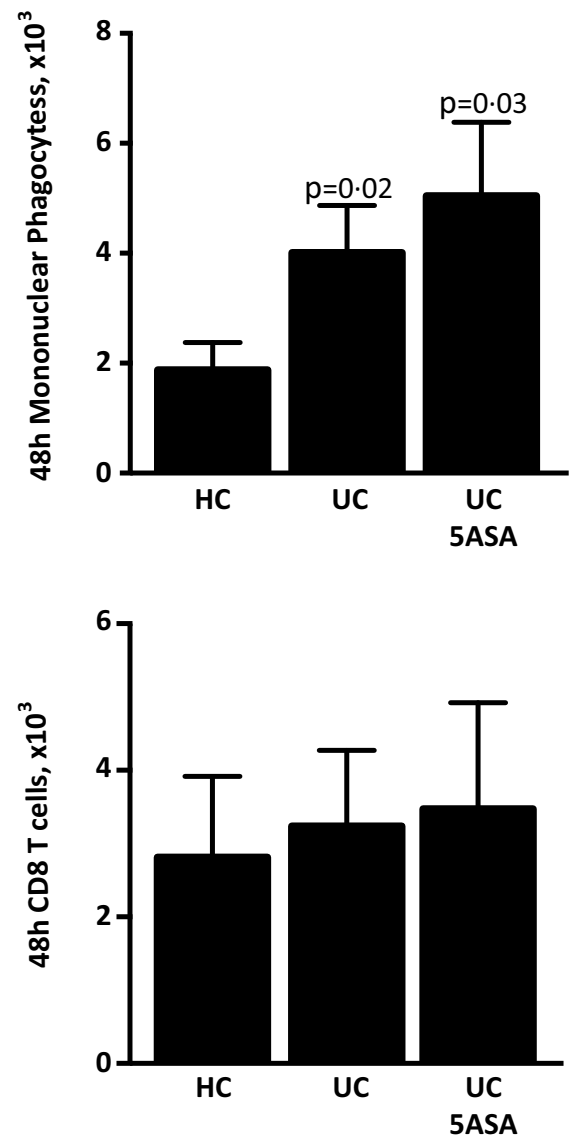
bioRxiv preprint doi: https://doi.org/10.1101/870139; this version posted December 10, 2019. The copyright holder for this preprint (which was not certified by peer review) is the author/funder, who has granted bioRxiv a license to display the preprint in perpetuity. It is made available under aCC-BY-NC-ND 4.0 International license. Supplementary Figure 2

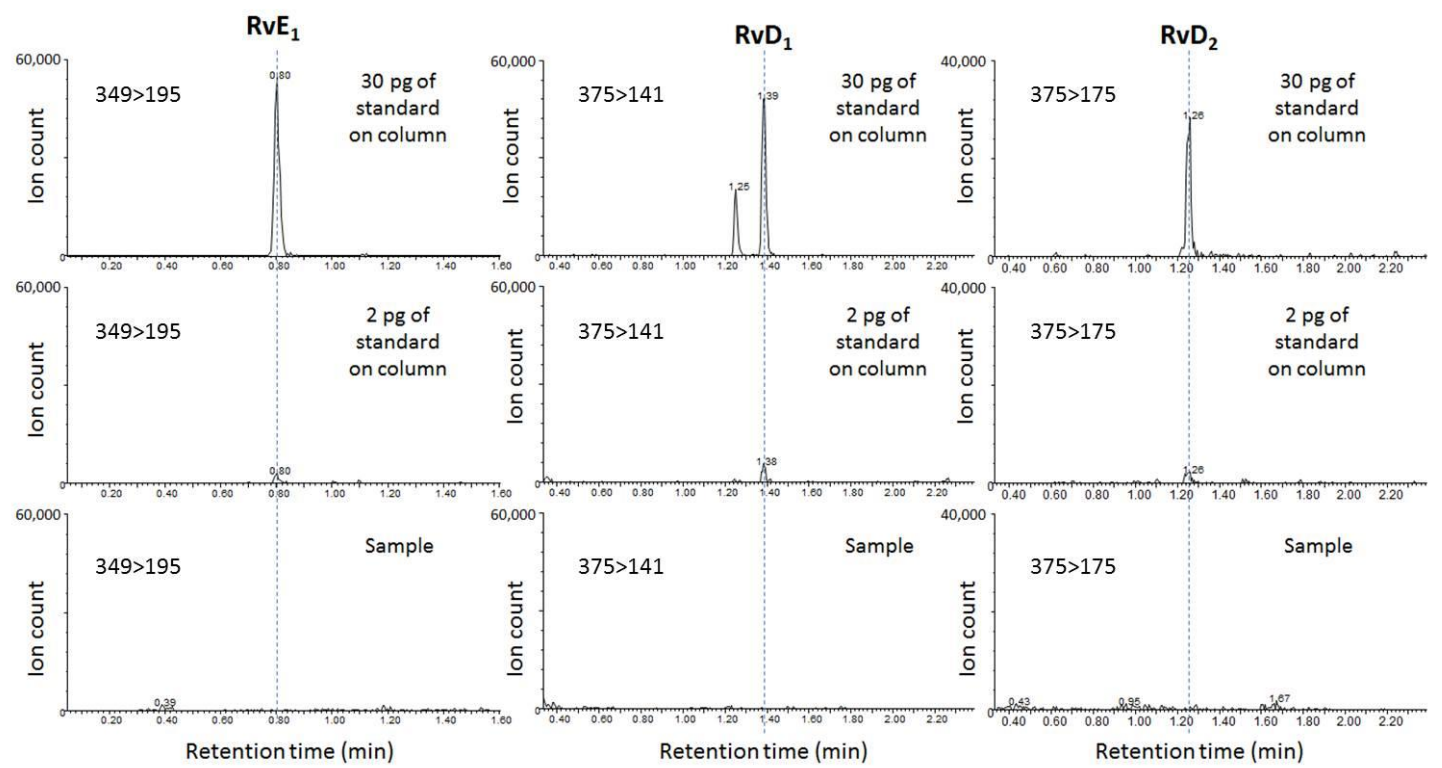




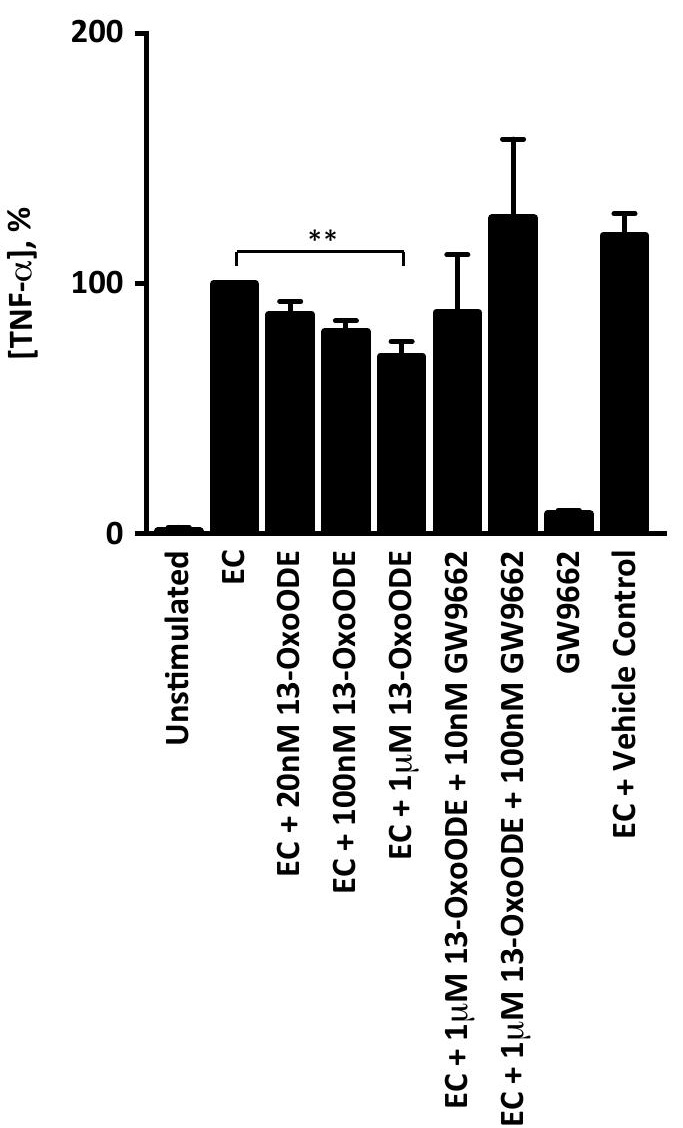


bioRxiv preprint doi: https://doi.org/10.1101/870139; this version posted December 10, 2019. The copyright holder for this preprint (which was not certified by peer review) is the author/funder, who has granted bioRxiv a license to display the preprint in perpetuity. It is made available

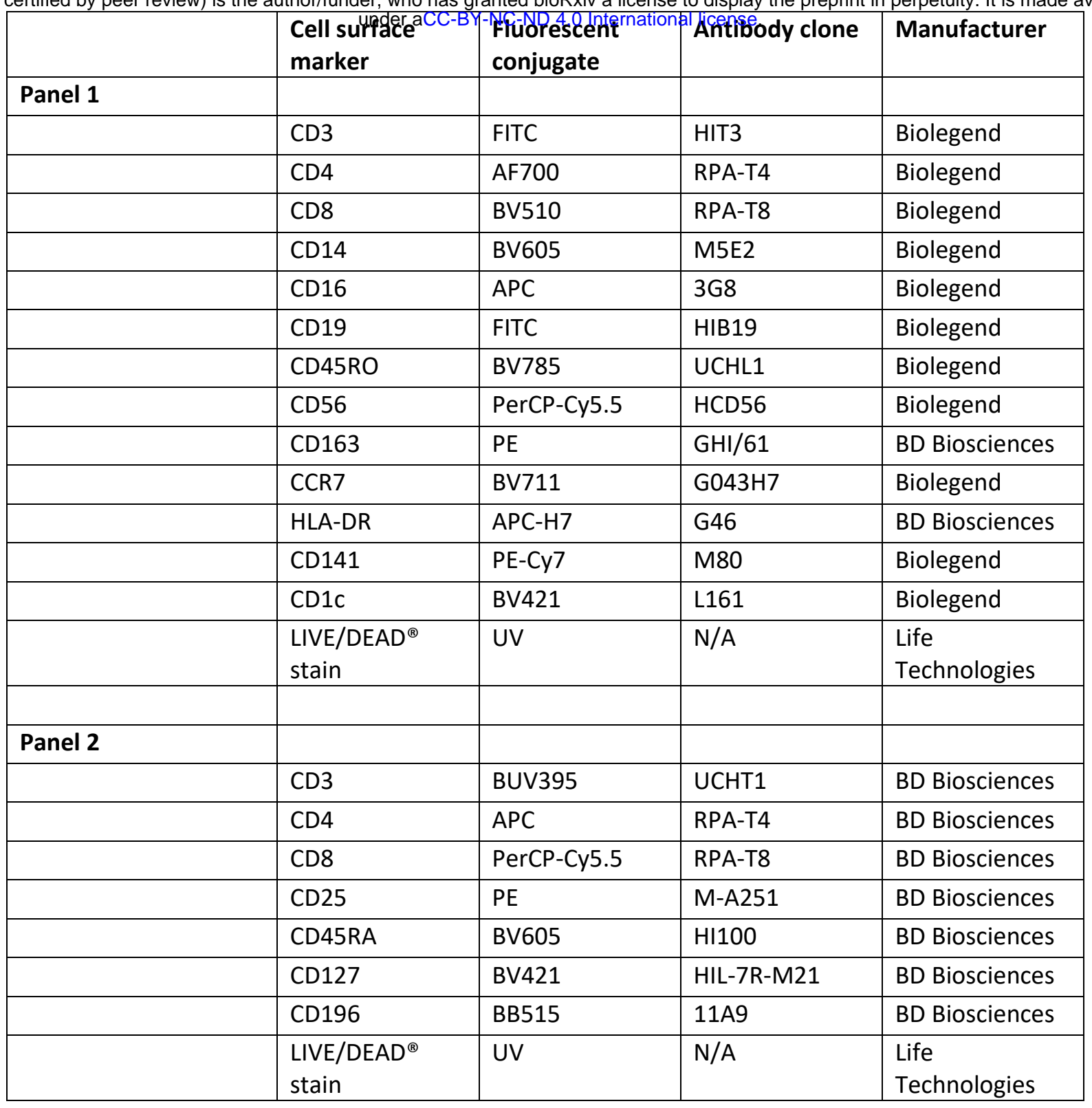

Supplementary Table 1 Antibody panel used in flow cytometry experiments 
bioRxiv preprint doi: https://doi.org/10.1101/870139; this version posted December 10, 2019. The copyright holder for this preprint (which was not certified by peer review) is the author/funder, who has granted bioRxiv a license to display the preprint in perpetuity. It is made available

\begin{tabular}{|c|c|c|c|c|}
\hline & \multicolumn{3}{|c|}{ 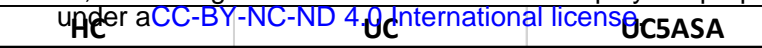 } & HC 5ASA \\
\hline \multicolumn{5}{|l|}{ Eicosanoids } \\
\hline PGD2 & $762 \pm 210$ & $977 \pm 233$ & $1046 \pm 261$ & $761 \pm 140$ \\
\hline PGE1 & $433 \pm 66$ & $724 \pm 199$ & $489 \pm 176$ & $959 \pm 408$ \\
\hline 13,14-dihydro-15-keto-PGE1 & $20 \pm 15$ & $130 \pm 55$ & $74 \pm 42$ & $52 \pm 52$ \\
\hline PGE2 & $6573 \pm 1282$ & $9407 \pm 1758$ & $11152 \pm 4811$ & $15336 \pm 10053$ \\
\hline 13,14-dihydro-15-keto-PGE2 & $1949 \pm 271$ & $5410 \pm 1229$ & $3306 \pm 816$ & $3234 \pm 1708$ \\
\hline D12 PGJ2 & $1009 \pm 111$ & $1294 \pm 252$ & $1192 \pm 221$ & $1669 \pm 371$ \\
\hline 15-deoxy-D12,14-PGJ2 & $210 \pm 69$ & $221 \pm 53$ & $164 \pm 54$ & $252 \pm 74$ \\
\hline PGF1 $\alpha$ & $469 \pm 68$ & $743 \pm 226$ & $399 \pm 106$ & $458 \pm 52$ \\
\hline 6-keto-PGF1 $\alpha$ & $2119 \pm 372$ & $3308 \pm 978$ & $3781 \pm 2059$ & $4150 \pm 3328$ \\
\hline PGF2 $\alpha$ & $7335 \pm 1110$ & $11951 \pm 3679$ & $7345 \pm 2098$ & $6093 \pm 1986$ \\
\hline 13,14-dihydro-15-keto-PGF2 $\alpha$ & 0 & $30 \pm 20$ & $41 \pm 28$ & 0 \\
\hline TXB2 & $5213 \pm 1295$ & $7904 \pm 1571$ & $8957 \pm 5593$ & $8243 \pm 3934$ \\
\hline \multicolumn{5}{|l|}{ Hydroxy lipids } \\
\hline 4-HDHA & $253 \pm 62$ & $383 \pm 47$ & $528 \pm 166$ & $1137 \pm 322$ \\
\hline 13-HDHA & $4 \pm 4$ & $36.55 \pm 24.21$ & 0 & 0 \\
\hline 14-HDHA & $161 \pm 65$ & $190 \pm 74$ & $63 \pm 48$ & $294 \pm 187$ \\
\hline 17-HDHA & 0 & 0 & $1055 \pm 1055$ & 0 \\
\hline 20-HDHA & $18 \pm 17$ & $6 \pm 6$ & $21 \pm 21$ & 0 \\
\hline 11,12-DHET & $160 \pm 31$ & $243 \pm 114$ & $143 \pm 40$ & $169 \pm 52$ \\
\hline 14,15-DHET & $140 \pm 30$ & $203 \pm 29$ & $219 \pm 23$ & $182 \pm 70$ \\
\hline 5-HETE & $37 \pm 24$ & $35 \pm 35$ & $116 \pm 62$ & $158 \pm 61$ \\
\hline 11-HETE & $1047 \pm 106$ & $1626 \pm 576$ & $2277 \pm 1619$ & $1743 \pm 1011$ \\
\hline 12-HETE & $8237 \pm 3071$ & $3790 \pm 641.5$ & $4136 \pm 946.1$ & $5401 \pm 1924$ \\
\hline 15-HETE & $2495 \pm 442$ & $2406 \pm 458$ & $2829 \pm 1876$ & $4161 \pm 1038$ \\
\hline 20-HETE & $410 \pm 155$ & $584 \pm 40$ & $922 \pm 711$ & $735 \pm 480$ \\
\hline 15-HETrE & $938 \pm 202$ & $1241 \pm 535$ & $543 \pm 238$ & $2370 \pm 454$ \\
\hline 11(12)-EET & 0 & $72 \pm 72$ & $154 \pm 154$ & $46 \pm 46$ \\
\hline 12-HEPE & $218 \pm 164$ & $17 \pm 17$ & $52 \pm 33$ & 0 \\
\hline 18-HEPE & $234 \pm 234$ & 0 & $100 \pm 63$ & 0 \\
\hline 9-HODE & $4744 \pm 409$ & $6180 \pm 1047$ & $4621 \pm 709$ & $5028 \pm 600$ \\
\hline 13-HODE & $10422 \pm 763$ & $16192 \pm 5145$ & $10590 \pm 1725$ & $13578 \pm 1697$ \\
\hline 9-OxOODE & $5056 \pm 668$ & $6382 \pm 1461$ & $5918 \pm 860$ & $4987 \pm 1208$ \\
\hline 13-OxoODE & $3605 \pm 733$ & $3931 \pm 1172$ & $3851 \pm 685$ & $2585 \pm 659$ \\
\hline 9-HOTrE & $732 \pm 135$ & $494 \pm 115$ & $449 \pm 181$ & $3411 \pm 751$ \\
\hline 13-HOTrE & $337 \pm 54$ & $244 \pm 87$ & $315 \pm 118$ & $664 \pm 139$ \\
\hline 9(10)-EpOME & $843 \pm 149$ & $1371 \pm 465$ & $1221 \pm 311$ & $1297 \pm 358$ \\
\hline 12(13)-EpOME & $1295 \pm 219$ & $1937 \pm 450$ & $1883 \pm 450$ & $2180 \pm 459$ \\
\hline Trans-EKODE & $1651 \pm 243$ & $17310 \pm 16341$ & $1105 \pm 139$ & $3060 \pm 869$ \\
\hline 9,10-DiHOME & $1026 \pm 84$ & $7460 \pm 5185$ & $1437 \pm 326$ & $1839 \pm 270$ \\
\hline 12,13-DiHOME & $1358 \pm 254$ & $11170 \pm 9035$ & $2047 \pm 495.9$ & $2017 \pm 380$ \\
\hline 19,20-DiHDPA & $1014 \pm 143$ & $763 \pm 117$ & $512 \pm 179$ & $483 \pm 29$ \\
\hline
\end{tabular}

Supplementary Table 2. Concentration ( $\mathrm{pg} / \mathrm{ml}$; mean \pm s.e.m.) of lipid mediators in $4 \mathrm{~h}$ blisters. 
bioRxiv preprint doi: https://doi.org/10.1101/870139; this version posted December 10, 2019. The copyright holder for this preprint (which was not certified by peer review) is the author/funder, who has granted bioRxiv a license to display the preprint in perpetuity. It is made available

\begin{tabular}{|c|c|c|c|c|}
\hline & \multicolumn{3}{|c|}{ 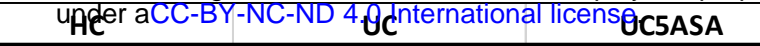 } & HC 5ASA \\
\hline \multicolumn{5}{|l|}{ Eicosanoids } \\
\hline PGD2 & $293 \pm 54$ & $579 \pm 179$ & $372 \pm 146$ & $227 \pm 48$ \\
\hline PGE1 & $150 \pm 24$ & $145 \pm 38$ & $117 \pm 53$ & $276 \pm 84$ \\
\hline 13,14-dihydro-15-keto-PGE1 & $6 \pm 4$ & $7 \pm 7$ & $6 \pm 6$ & $23 \pm 23$ \\
\hline PGE2 & $1168 \pm 164$ & $1426 \pm 256$ & $1161 \pm 272$ & $1570 \pm 348$ \\
\hline 13,14-dihydro-15-keto-PGE2 & $148 \pm 27$ & $251 \pm 49$ & $216 \pm 49$ & $150 \pm 26$ \\
\hline D12 PGJ2 & $704 \pm 121$ & $670 \pm 111$ & $727 \pm 124$ & $584 \pm 92$ \\
\hline 15-deoxy-D12,14-PGJ2 & $171 \pm 58$ & $103 \pm 24$ & $118 \pm 26$ & $83 \pm 12$ \\
\hline PGF1 $\alpha$ & $135 \pm 29$ & $104 \pm 24$ & $83 \pm 31$ & $75 \pm 56$ \\
\hline 6-keto-PGF1 $\alpha$ & $138 \pm 27$ & $94 \pm 16$ & $66 \pm 22$ & $89 \pm 30$ \\
\hline PGF2 $\alpha$ & $1331 \pm 215$ & $1214 \pm 204$ & $1184 \pm 391$ & $521 \pm 76$ \\
\hline 13,14-dihydro-15-keto-PGF2 $\alpha$ & 0 & 0 & 0 & 0 \\
\hline TXB2 & $742 \pm 190$ & $669 \pm 197$ & $271 \pm 107$ & $395 \pm 66$ \\
\hline \multicolumn{5}{|l|}{ Hydroxy lipids } \\
\hline 4-HDHA & $251 \pm 52$ & $245 \pm 123$ & $328 \pm 113$ & $515 \pm 89$ \\
\hline 13-HDHA & 0 & $18 \pm 18$ & 0 & 0 \\
\hline 14-HDHA & $111 \pm 34$ & $53 \pm 38$ & $14 \pm 14$ & $87 \pm 53$ \\
\hline 17-HDHA & $79 \pm 79$ & $563 \pm 371$ & 0 & 0 \\
\hline 20-HDHA & $73 \pm 36$ & $58 \pm 38$ & $44 \pm 44$ & 0 \\
\hline 11,12-DHET & $159 \pm 17$ & $129 \pm 28$ & $167 \pm 24$ & $209 \pm 35$ \\
\hline 14,15-DHET & $231 \pm 10$ & $225 \pm 40$ & $244 \pm 43$ & $211 \pm 61$ \\
\hline 5-HETE & $23 \pm 15$ & $42 \pm 27$ & $42 \pm 28$ & $139 \pm 63$ \\
\hline 11-HETE & $225 \pm 22$ & $165 \pm 30$ & $176 \pm 35$ & $129 \pm 26$ \\
\hline 12-HETE & $4970 \pm 1895$ & $2127 \pm 444$ & $2038 \pm 354$ & $3465 \pm 1349$ \\
\hline 15-HETE & $815 \pm 74$ & $933 \pm 445$ & $497 \pm 173$ & $670 \pm 130$ \\
\hline 20-HETE & $161 \pm 84$ & $234 \pm 149$ & 0 & $169 \pm 169$ \\
\hline 15-HETrE & $468 \pm 68$ & $594 \pm 191$ & $335 \pm 74$ & $529 \pm 149$ \\
\hline 11(12)-EET & $103 \pm 50$ & $21 \pm 21$ & $46 \pm 30$ & $50 \pm 33$ \\
\hline 12-HEPE & $169 \pm 92$ & $13 \pm 13$ & $5 \pm 5$ & $40 \pm 24$ \\
\hline 18-HEPE & $38 \pm 25$ & 0 & 0 & 0 \\
\hline 9-HODE & $3950 \pm 513$ & $4501 \pm 517$ & $4205 \pm 739$ & $2653 \pm 612$ \\
\hline 13-HODE & $9210 \pm 1122$ & $10221 \pm 1613$ & $9679 \pm 1542$ & $6491 \pm 1310$ \\
\hline 9-OxoODE & $3362 \pm 404$ & $4387 \pm 238$ & $6023 \pm 978$ & $2005 \pm 170$ \\
\hline 13-OxoODE & $1862 \pm 319$ & $2899 \pm 406$ & $3955 \pm 507$ & $1442 \pm 200$ \\
\hline 9-HOTrE & $532 \pm 121$ & $625 \pm 182$ & $482 \pm 200$ & $1453 \pm 371$ \\
\hline 13-HOTrE & $288 \pm 34$ & $192 \pm 60$ & $158 \pm 68$ & $387 \pm 86$ \\
\hline 9(10)-EpOME & $1386 \pm 378$ & $1069 \pm 256$ & $1462 \pm 337$ & $1632 \pm 219$ \\
\hline 12(13)-EpOME & $1719 \pm 452$ & $1451 \pm 319$ & $1918 \pm 220$ & $1562 \pm 173$ \\
\hline Trans-EKODE & $964 \pm 142$ & $1195 \pm 145$ & $1340 \pm 332$ & $723 \pm 218$ \\
\hline 9,10-DiHOME & $1231 \pm 232$ & $1326 \pm 206$ & $1566 \pm 383$ & $1728 \pm 272$ \\
\hline 12,13-DiHOME & $1630 \pm 317$ & $1444 \pm 395$ & $1979 \pm 574$ & $2078 \pm 480$ \\
\hline 19,20-DiHDPA & $694 \pm 85$ & $733 \pm 129$ & $759 \pm 208$ & $347 \pm 63$ \\
\hline
\end{tabular}

Supplementary Table 3. Concentration (pg/ml; mean \pm s.e.m.) of lipid mediators in $48 \mathrm{~h}$ blisters. 